\title{
An Iterative Least Squares Error Method with Resampling for Frequency Tracking in Power System
}

\author{
LI Hui ${ }^{1, a}$, CAO Chun-Jie ${ }^{2, b^{*}}$ and YUAN Liang ${ }^{3, c}$
}

${ }^{1}$ Room 309 of School of Information Science and Technology, Hainan University, 58 Renmin Ave, Haikou 570228, P. R. China

${ }^{2}$ Room 223 of School of Information Science and Technology, Hainan University, 58 Renmin Ave, Haikou 570228, P. R. China

${ }^{3}$ Haisum Engineering CO.,LTD., No. 21 of Baoqing Road, Shanghai 200031, P. R. China

ahitlihui1112@163.com, ${ }^{b}$ lianghuicoap@163.com, cyuanliang@haisum.com

Keywords: Power System, Frequency Tracking, Least Squares Error, Resampling

Abstract. Phase angle and amplitude of a phasor can be provided by method of least squares error (LSE). LSE adopted iteratively is able to track the frequency and amplitude of power system in steady states and in kinds of non-steady ones. Iterative LSE with resampling is analyzed in conditions of off-nominal input, nominal input with harmonics and decaying direct current (DC) offset and additive Gaussian white noise.Performance of resampling LSE is compared with traditional LSE. Resampling LSE has better performance than the traditional one in frequency tracking ability and can provide less mean square error.

\section{Introduction}

Frequency is one of the most important situation variables and operation parameter in power system. It is a good indicator of integrity of a power system facing separation and islanding. Phasor measurement unit (PMU) provides an indication of lost generation, as an example, a frequency drop of $0.1 \mathrm{~Hz}$ is typical in the Western Electricity Coordinating Council for $800 \mathrm{MW}$ generation loss [1]. In the past several decades researchers have paid much attention to frequency measurement and analysis in power engineering. Types of frequency estimation methods have been reported, such as zero crossing [2,3], demodulation technique [2],Newton algorithm[4], Kalman filter [5 7], prony approach [8], artificial neural network [9], etc. in time-domain. In the transform-domain, the discrete Fourier transform (DFT)/fast Fourier transform $[10,11]$ is widely adopted as well as discrete wavelet transform [12 15].

Frequency can be calculated by least squares error (LSE) with a pseudo-inverse of a matrix that is determined by a frequency $\omega_{0}$ and samples at time $t_{m}\left(m=1,2, \ldots, M\right.$, samples at $t_{1} \sim t_{M}$ constituting a data window) of the input signal[16]. Using LSE iteratively is able to track system frequency sample-by-sample. Its estimation accuracy is determined by the number of iterations per sample interval and by computation ability of hardware in real-time application.

\section{Frequency Estimation by Least Square Error Method}

Traditional LSE Method. Suppose the nominal voltage or current signal of a power system is

$$
s(t)=\sum_{i=1}^{H} A_{i} \cos \left(\omega_{i} t+\varphi_{i}\right)(-\infty<t<\infty)
$$

where $H$ is the order of highest harmonic, and $A_{i}, \omega_{i}=i \times \omega_{i}, \varphi_{i}$ are the amplitude, angular frequency and initial phase angle of each harmonic. According to rule of Nyquist, least sampling frequency $f_{s} \geq 2 \times H f_{1}$ should be chosen to eliminate aliasing. And thanks to low pass filter, components with frequency higher than $H f_{1}$ are filtered, so we have

$V=A \cdot X$ 
where $V=\left[s\left(t_{1}\right), s\left(t_{2}\right), \ldots, s\left(t_{2 H}\right)\right]^{T}$, in which $s\left(t_{1}\right), s\left(t_{2}\right)$ and $s\left(t_{2 H}\right)$ are the samples taken at time $t_{1}, t_{2}$ and $t_{2 H}$ (at least $2 H$ samples are taken); $X=\left[X_{1}, X_{2}, \ldots, X_{2 H-1}, X_{2 H}\right]^{T}$, in which $X_{1}=A_{1} \cos \varphi_{1}, X_{2}=A_{1} \sin \varphi_{1}, \ldots$, and $X_{2 H-1}=A_{H} \cos \varphi_{H}, X_{2 H}=A_{H} \sin \varphi_{H} ;$ and the matrix $A=\left[\begin{array}{ccccc}a_{11} & a_{12} & \mathrm{~L} & a_{1 \_2 H-1} & a_{1 \_2 H} \\ \mathrm{M} & \mathrm{M} & \mathrm{O} & \mathrm{M} & \mathrm{M} \\ a_{2 H_{-} 1} & a_{2 H_{-} 2} & \mathrm{~L} & a_{2 H_{-} 2 H-1} & a_{2 H_{-} 2 H}\end{array}\right]$, in which $a_{11}=\sin \left(\omega_{1} t_{1}\right), a_{12}=\cos \left(\omega_{1} t_{1}\right), a_{13}=\sin \left(\omega_{2} t_{1}\right)$, $a_{14}=\cos \left(\omega_{2} t_{1}\right) \quad, \ldots, \quad a_{1 \_2 H-1}=\sin \left(\omega_{H} t_{1}\right) \quad, \quad a_{1 \_2 H}=\cos \left(\omega_{H} t_{1}\right) \quad, \ldots, \quad$ and $\quad a_{2 H \_1}=\sin \left(\omega_{1} t_{2 H}\right)$, $a_{2 H_{-} 2}=\cos \left(\omega_{1} t_{2 H}\right), a_{2 H_{-} 3}=\sin \left(\omega_{2} t_{2 H}\right), a_{2 H_{-} 4}=\cos \left(\omega_{2} t_{2 H}\right), \ldots, \quad a_{2 H_{-} 2 H-1}=\sin \left(\omega_{H} t_{2 H}\right)$, $a_{2 H_{2} 2 H}=\cos \left(\omega_{H} t_{2 H}\right)$. When a fault or a disturbance occurs, the current signal consists of exponentially decaying DC offsets in electrical power system. The decaying rates depend on the time constants determined by the inductive reactance to resistance ratio (X/R ratio) of the system. The larger the $\mathrm{X} / \mathrm{R}$ ratio, the slower the DC component decays. Signal with the nominal component and a decaying DC offset is represented as

$$
s(t)=A_{1} \cos \left(\omega_{1} t+\varphi_{1}\right)+A_{d c} e^{-t / \tau}\left(0 \leq t \leq T_{D C}\right)
$$

where $A_{d c}$ and $\tau$ are the amplitude and time constant of the decaying DC offset component. $T_{D C}$ is the effecting period of decaying DC offset, since the decaying DC offset exists only in several cycles and then disappears. It is a non-periodic signal and its frequency spectrum encompasses all the frequencies which cannot be removed by anti-aliasing low pass filter. Matrix $A$ has to be rewritten according to input signal as $\left[\begin{array}{rrrr}a_{11} & a_{12} & \mathrm{~L} & a_{15} \\ a_{21} & a_{22} & \mathrm{~L} & a_{25} \\ \mathrm{M} & \mathrm{M} & \mathrm{O} & \mathrm{M} \\ a_{m 1} & a_{m 2} & \mathrm{~L} & a_{m 5}\end{array}\right]$, where $a_{m 1}=1, a_{m 2}=t_{m}, a_{m 3}=t_{m}^{2}, a_{m 4}=\sin \left(\omega_{1} t_{m}\right), a_{m 5}=\cos \left(\omega_{1} t_{m}\right)$ at time $t_{m}(m \geq 5)$ for iterative LSE. In order to obtain $X$, we have $X=\left[A^{T} A\right]^{-1} A^{T} V=A^{+} V$

We get phasor with amplitude $Y^{1}$ and phase angle $\theta^{1}$

$Y\left(t_{1}\right)=Y^{1} e^{j \theta^{1}}$

where

$\theta^{1}=\operatorname{atan}\left[Y\left(t_{1}\right)_{\text {image }} / Y\left(t_{1}\right)_{\text {real }}\right]$

where atan is the inverse tangent function. $Y\left(t_{1}\right)_{\text {image }}$ and $Y\left(t_{1}\right)_{\text {real }}$ are the imaginary part and real part of phasor $Y\left(t_{1}\right)$. And $Y^{1}=\sqrt{X_{1}^{2}+X_{2}^{2}}$. In order to get $Y\left(t_{2}\right)$, we have to sample the input signal at time $t_{2}$ and $t_{3}$, whose time interval is $T_{s}$. The data window to get $Y\left(t_{2}\right)$ is shifted by one $T_{s}$ from that of $Y\left(t_{1}\right)$. So the angular frequency is calculated by $f_{1}=\left(\theta^{2}-\theta^{1}\right) /\left(2 \pi T_{s}\right)$. Generally we have frequency at $t_{m}$

$f_{1}^{m}=\left(\theta^{m+1}-\theta^{m}\right) /\left(2 \pi T_{s}\right)$

Traditional LSE algorithm uses fixed sampling frequency of $720 \mathrm{~Hz}$ as specified in [16]. However, frequency of the input may change under different conditions, such as off-nominal signal in kinds of transient states or under sudden step changes. In these circumstances one data window may contain non-integer number of samples that leads to spectrum leakage and error of estimation. If we change the rate of sampling according to previous estimated frequencies, there may be integer number of sample in one data window, and then the error of frequency would be minimized. Also the nominal signal may be polluted by harmonics, inter-harmonics, decaying DC offsets, additive Gaussian with noise, etc. Window-based data acquiring process is shown in Figure 1.

Frequency Estimation by Iterative Resampling-LSE Method. Suppose frequency of input signal is an off-nominal one. By Equation (7), we can estimate the frequency iteratively by resetting the matrix $A$ with different estimated frequency $f^{\prime}$. In reality, sampling rate is set properly to fulfill 


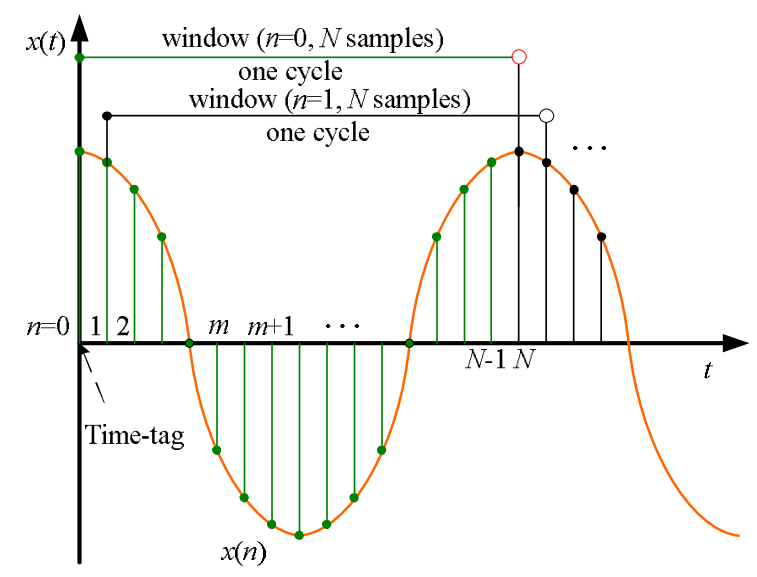

Fig. 1 Illustration of shifting windows of a cosine waveform with time-tag at starting time of $t=0 \mathrm{~s}$ the requirement of Nyquist's rule and the criterion of sampling in [4]. Secondly, an over-determined linear equation set is built to give a more precise estimation with more samples. Steps of iterative LSE method are:

Step 1: Sample the signal with frequency $f_{s}$ and form a data window.

Step 2: Obtain matrix $A$ according to sampling frequency at time $t_{m}, m=1,2, \ldots, M$, where $M$ is the length of data window. $M$ is bigger than length of vector $X$ to construct an over-determined linear equation set.

Step 3: Obtain $X$ according to Equation (4).

Step 4: Obtain phase angle of phasor $Y\left(t_{1}\right)$ and $Y\left(t_{2}\right)$ by Equation (6) and then estimated frequency $f^{\prime}$ by Equation (7). Number of iteration is 1 .

Step 5: Resample the input signal and reset the matrix $A$ according to estimated frequency $f^{\prime}$ at time $t_{m}, m=1,2, \ldots, M$. Repeat steps $1 \sim 4$ and get $f^{\prime \prime}$. Number of iteration is 2 .

Step 6: If $\left|f^{\prime}-f^{\prime \prime}\right| \leq \delta$, iteration process converge, where $\delta$ is a preset minimal number; and also if number iteration approaches the maximal number $N_{\text {ite_max }}$, iteration process stops.

In order to track the frequency sample-by-sample, we have to use the process of the iterative LSE per sampling time interval. The initial frequency to get matrix $A$ in the following interval is also the estimated one of previous interval. Number of iterations per sampling interval, limited by computation ability of a digital processor, influences frequency tracking ability and convergence speed of the estimations process. Method of LSE adopts iteration process to get the off-nominal frequency more and more precisely. More iteration is accomplished during one time interval, more accurate we get, but more time is consumed.

\section{Frequency Tracking Using Resampling LES}

Suppose the input signal contains a $3^{\text {rd }}$ order harmonic and a decaying DC offset lasting in several cycles at the beginning of simulation as shown in Equation (8). Parameters used in simulation are listed in Table I. We can design the matrix $A$ with the help of Taylor expansion as $A=\left[\begin{array}{rrrr}a_{11} & a_{12} & \mathrm{~L} & a_{17} \\ a_{21} & a_{22} & \mathrm{~L} & a_{27} \\ \mathrm{M} & \mathrm{M} & \mathrm{O} & \mathrm{M} \\ a_{m 1} & a_{m 2} & \mathrm{~L} & a_{m 7}\end{array}\right]$, where $a_{m 1}=1, a_{m 2}=t_{m}, a_{m 3}=t_{m}^{2}, a_{m 4}=\sin \left(\omega_{1} t_{m}\right), a_{m 5}=\cos \left(\omega_{1} t_{m}\right), a_{m 6}=\sin \left(\omega_{3} t_{m}\right), a_{m 7}=\cos \left(\omega_{3} t_{m}\right)$ at time $t_{m}(m \geq 7) ; \omega_{3}=2 \pi f_{3}$; and $X=\left[X_{1}, X_{2}, \ldots, X_{7}\right]^{T}$, where $X_{1}=A_{d c}, X_{2}=-A_{d c} / \tau, X_{3}=A_{d c} /\left(2 \tau^{2}\right)$, $X_{4}=A_{1} \cos \varphi_{1}, X_{5}=A_{1} \sin \varphi_{1}, X_{6}=A_{3} \cos \varphi_{3}, X_{7}=A_{3} \sin \varphi_{3}$.

At first, we choose the best sampling rate and data window for LSE algorithms, since the performance of these algorithms are highly connected with them. Former studies show that lower 
sampling rate and longer data window help to obtain a better result [16]. $f_{\text {input }}$ denotes the frequency of input signal.

Table I Basic parameters utilized in simulation

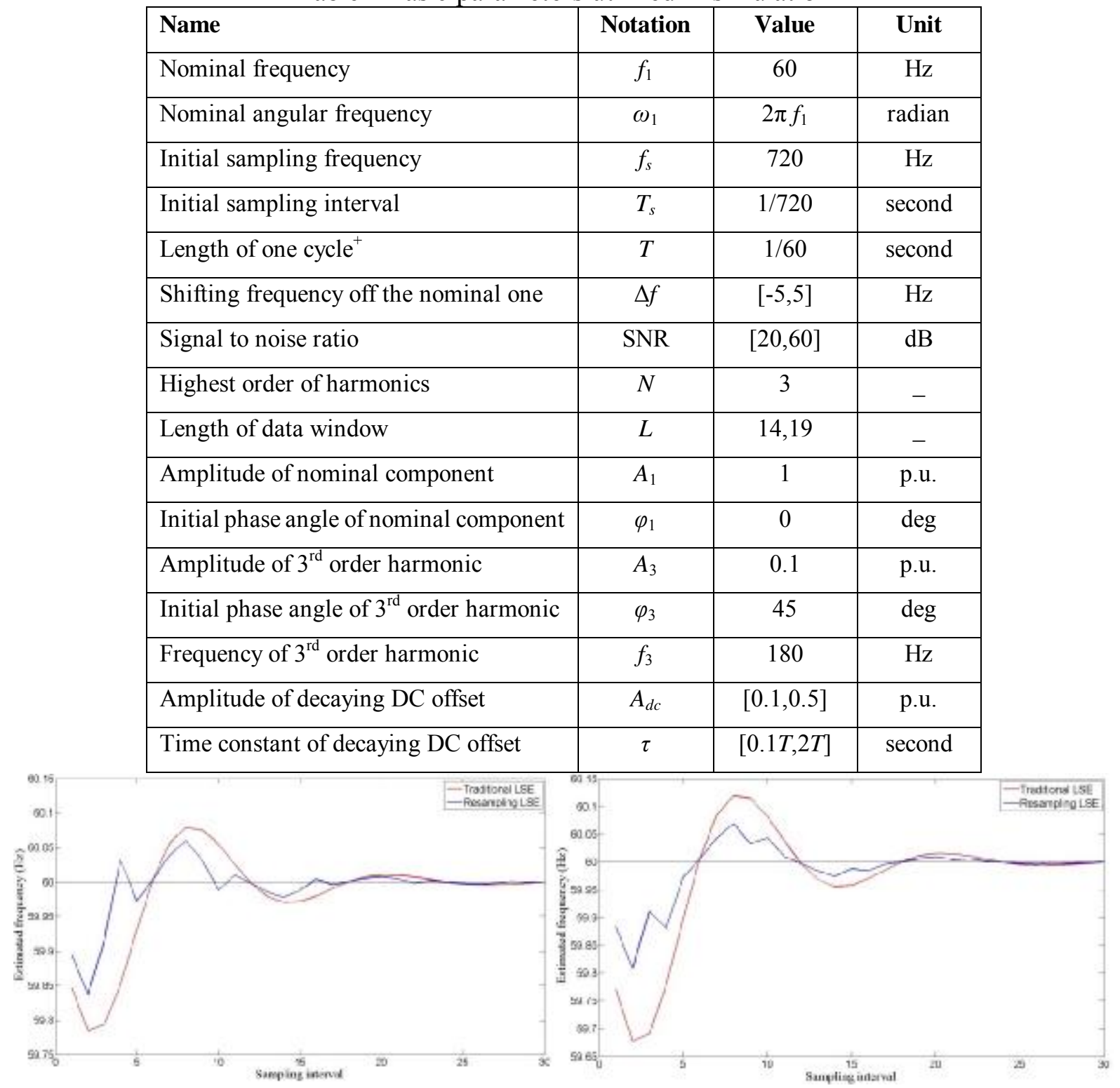

(a) Number of iteration $N_{i t e}=2$ in each sampling interval

(b) Number of iteration $N_{i t e}=3$ in each sampling interval Fig.3 Performance of traditional and resampling LSE methods with nominal input frequency (Initial sampling rate $f_{s}=720 \mathrm{~Hz}$, data window $L=14, f_{\text {input }}=60 \mathrm{~Hz}$, first 30 estimated frequencies are plotted)

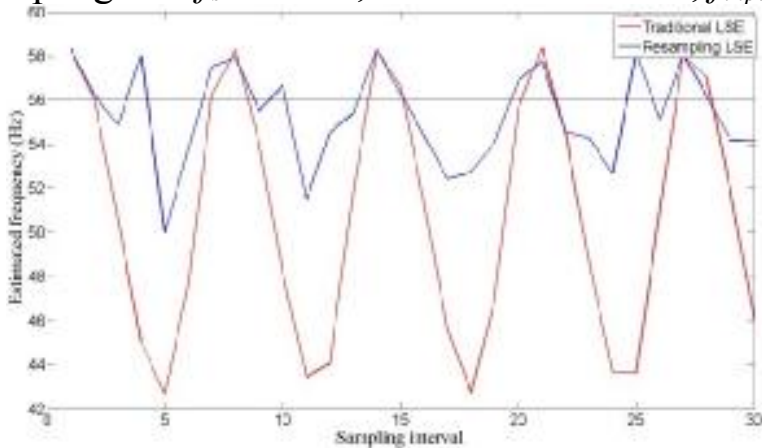

(a) $f_{\text {input }}=55 \mathrm{~Hz}$

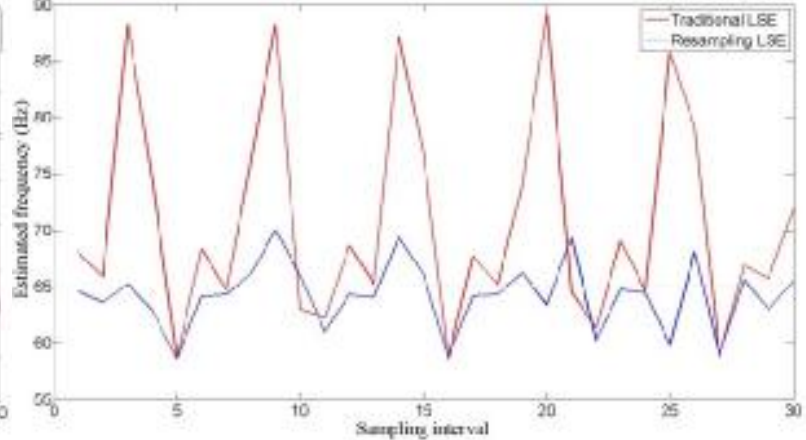

(b) $f_{\text {input }}=65 \mathrm{~Hz}$

Fig.4 Performance of traditional and resampling LSE methods with off-nominal input frequency (Initial sampling rate $f_{s}=720 \mathrm{~Hz}$, data window $L=14$, Number of iteration=2, first 30 estimated frequencies are plotted) 
In Figure 3, we find that the number of iterations during each sampling interval is set to be 2 or 3, which is good enough to acquire a high accuracy for resampling LSE, and comparing with the traditional one, the resampling LSE method tends to converge more robustly. In Figure 4, we know that tracking accuracy of resampling LSE in higher than that of the traditional one. The dynamic range of resampling LSE is smaller than that of the traditional one. Figure 5 shows the mean square error (MSE) of two LSE methods with different variables such as time constant and amplitude of decaying DC offset component. When the amplitude of decaying DC offset is 0.2 p.u. and the time constant is $0.1 T$, the MSE are 1.2106 and 0.5222 for traditional LSE and resampling LSE respectively.

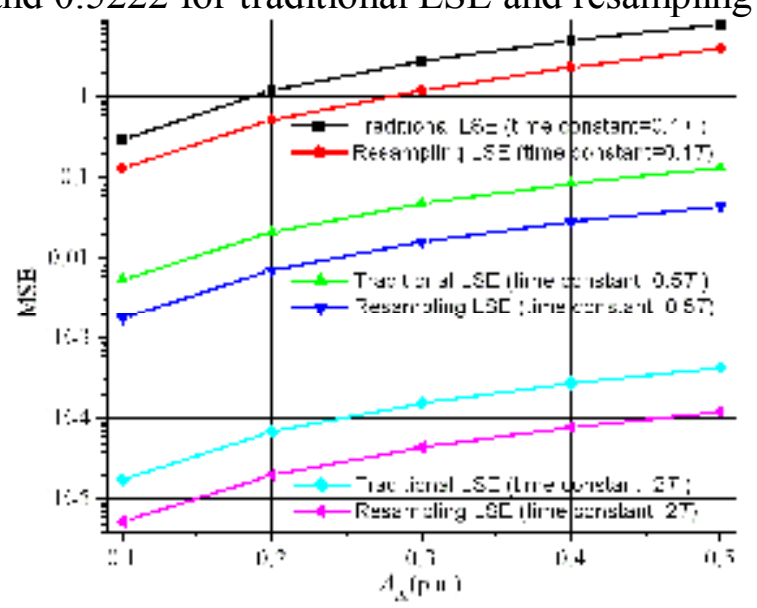

Fig. 5 Performance of traditional and resampling LSE methods with different decaying DC offset (Initial sampling rate $f_{s}=720 \mathrm{~Hz}$, data window $L=14$, Number of iteration $N_{\text {ite }}=2, f_{\text {input }}=60 \mathrm{~Hz}$, first 30

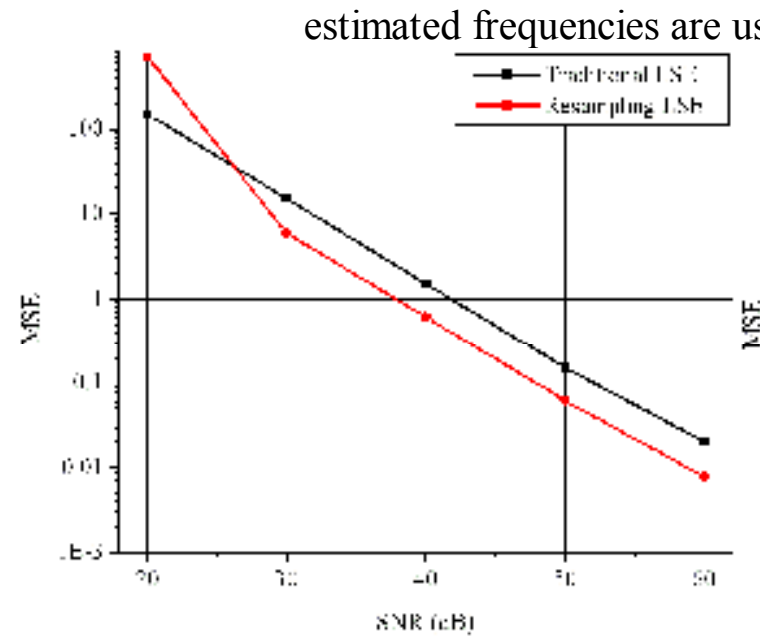

(a) Number of iteration $N_{i t e}=2$

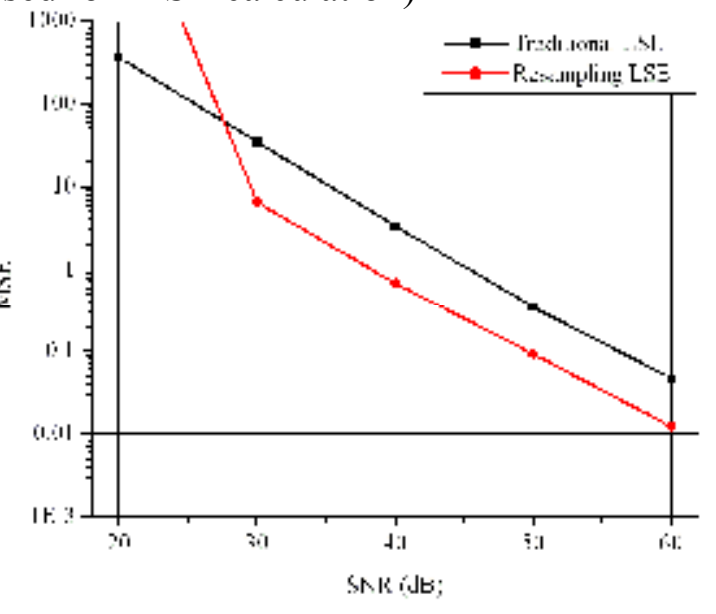

(b) Number of iteration $N_{\text {ite }}=3$

Fig. 6 Performance of traditional and resampling LSE methods with noise (Initial sampling rate $f_{s}=720 \mathrm{~Hz}$, data window $L=14, f_{\text {input }}=60 \mathrm{~Hz}$, first 30 estimated frequencies are used for MSE calculation and averaged in 1000 times)

Figure 6 tell us that resampling LSE does not work well when signal to noise ratio (SNR) is less than $25 \sim 30 \mathrm{~dB}$. In the practice of power system, SNR is always higher than $30 \mathrm{~dB}$. So Gaussian white noise could not do too much harm to resampling LSE in practice.

\section{Summary}

We proposed a method of resampling LSE whose performance was analyzed and compared with the traditional one, when the incoming signal containing harmonics and decaying DC offset. The key point of resampling LSE is that it resamples the input signal according to frequency calculated in previous sampling interval, and the iterations processes are accomplished in each interval which is limited by the computation ability of digital signal processor chips. Iterative LSE can track the nominal frequency and the off-nominal one in presence of additive Gaussian white noise with harmonics. The resampling one 
is less sensitive to DC offset than traditional one. Resampling LSE shows better performance of frequency tracking comparison with the old one with less MSE and better performance in dynamic states of a power system.

\section{Acknowledgements}

The authors are greatly indebted to the anonymous referee, whose comments significantly improved the manuscript. This paper is supported by NSF of China under contract of 61071128 .

\section{References}

[1] North American Electric Reliability Corporation, Real-time application of synchrophasors for improving reliability, http://www.smartgrid.gov, Oct. 2010

[2] M. M. Begovic, P. M. Djuric, S. Dunlap, A. G. Phadke, Frequency tracking in power networks in the presence of harmonics, IEEE Transactions on Power Delivery, 8(1993) 480-486

[3] C. T. Nguyen, K. Srinivasan, A new technique for rapid tracking of frequency deviation based on level crossings, IEEE Transactions on Power Apparatus and Systems, 103(1984) 2230-2236

[4] V. V. Terzija, M. B. Djuric, B. D. Kovacevic, Voltage phasor and local system frequency estimation using Newton type algorithm, IEEE Transactions on Power Delivery, 9(1994) 1368-1374

[5] M. S. Sachdev, H. C. Wood, N. G. Johnson, Kalman filtering applied to power system measurements for relaying, IEEE Transactions on Power Apparatus and Systems, 104(1985) 3565-3573

[6] A. Routray, A. K. Pradhan, K. P. Rao, A novel Kalman filter for frequency estimation of distorted signals in power systems, IEEE Transactions on Instrumentation and Measurement, 51(2002) 469-479

[7] E. M. Siavashi, S. Afshania, M. T. Bina, M. K. Zadeh, M. R. Baradar, Frequency estimation of distorted signals in power systems using particle extended Kalman filter, $2^{\text {nd }}$ International Conference on Power Electronics and Intelligent Transportation System, 19-20 Dec., 1(2009) 174-178

[8] T. Lobos, J. Rezmer, Real-time determination of power system frequency, IEEE Transactions on Instrument and Measurement, 46(1997) 877-881

[9] R. Vianello, M. O. Prates, C. A. Duque, A. S. Cequeira, P. M. da Silveira, P. F. Ribeiro, New phasor estimator in the presence of harmonics, DC-offset and interharmonics, $14^{\text {th }}$ International Conference on Harmonics and Quality of Power, 26-29 Sept., (2010) 1-5

[10] J. Z. Yang, C. W. Liu, A new family of measurement technique for tracking voltage phasor, local system frequency, harmonics and DC offset, IEEE Power Engineering Society Summer Meeting, 16-20 July, 3(2000) 1327-1332

[11] B. Zeng, Z. S. Teng, Y. L. Cai, S. Y. Guo, B. Y. Qing, Harmonic phasor analysis based on improved FFT algorithm, IEEE Transactions on Smart Grid, 2(2011) 51-59

[12] O. Chaari, M. Meunier, F. Brouaye, Wavelet: a new tool for the resonant grounded power distribution systems relaying, IEEE Transactions on Power Delivery, 11(1996)1301-1308

[13] S. J. Huang, C. T. Hsieh, C. L. Huang, Application of Morlet wavelet to supervise power system disturbances, IEEE Transactions on Power Delivery, 14(1999) 235-243

[14] X. N. Lin, H. Zhang, P. Liu, O. P. Malik, Wavelet based scheme for detection of torsional oscillation, IEEE Transactions on Power System, 17(2002) 1096-1101

[15] J. F. Ren, M. Kezunovic, Real-time power system frequency and phasors estimation using iterative wavelet transform, IEEE Transactions on Power Delivery, 26(2011) 1392-1402

[16] M. S. Sachdev, M. A. Baribeau, A new algorithm for digital impedance relays, IEEE Transactions on Power Apparatus and Systems, 98(1979) 2232-2240 